\title{
A Generalised Geometry and Intensity Based Partial Volume Correction for Magnetic Resonance Images
}

\author{
F. Belloํ, A.C.F. Colchester ${ }^{1}$, S.A. Röll ${ }^{2}$ \\ 1 Neurosciences Medical Image Analysis Group, Kent Institute of Medicine and \\ Health Sciences, University of Kent at Canterbury, Canterbury Kent CT2 7NZ, UK \\ f.bello@ukc.ac.uk \\ 2 Tomography Group, University of Bremen, Germany
}

\begin{abstract}
Segmentation of objects of interest in magnetic resonance imaging is a necessary procedure for volumetric calculations. However, these direct measurements tend to be inaccurate due to the intrinsic MRI partial volume effects. In this paper, a generalised method for correcting these effects based on the geometry and grey level intensity of the segmented objects is presented. This method is independent of the segmentation strategy used to extract the objects of interest. An evaluation is presented for three different segmentation methods and it is shown that the proposed generalised partial volume correction can improve the volume estimation of all three methods.
\end{abstract}

\section{Introduction}

An important problem of volumetric measurements in magnetic resonance imaging (MRI) is partial volume (PV) effects. Since a finite volume is imaged with each slice, more than a single tissue type is likely to be included in each voxel resulting in the PV averaging of tissue. In the case of segmented objects, it is reasonable to consider only the $\mathrm{PV}$ effects at or near the extracted boundaries where averaging between the object and background takes place. This consideration is based on the assumption that all of the non-boundary or interior voxels of the object are fully 'occupied' by the same tissue class and that the determined boundaries are as close as possible to the real object boundaries. Thus, correcting for PV effects implies determining the percentage of object and background present in the voxels at or near the object boundaries. For wide applicability, it is important to be able to perform a systematic PV correction that is independent of the segmentation method.

The main motivation of the present work is the volume estimation of lesions present in MR brain scans of multiple sclerosis (MS) patients. Lesion volume is a widely used measure and its accurate determination is desirable. Segmentation of MS lesions with correction for PV averaging effects would represent a significant improvement in the accurate determination of lesion volumes and in the ultimate goal of establishing a correlation between lesion volumes and clinical disability. 
The remainder of this paper is organised as follows: Section 2 gives a brief review of related work. Section 3 discusses the basic concepts of the geometry and intensity based PV correction method introduced in [1] and presents its generalisation. Section 4 describes the synthetic lesions used to evaluate the proposed generalised PV correction method, the three different segmentation techniques employed and the results obtained. These results are discussed in Section 5. The final section presents the conclusions and further work contemplated.

\section{Related Work}

Most of the recent MRI partial volume correction work found in the literature is based on multi-spectral images. Soltanian-Zadeh et al [2] presented a feature space analysis method including a correction for PV effects. A multi-spectral statistical analysis of PW tissue classification based on Markovian Random Fields was discussed by Choi et al [3]. The effectiveness of various fuzzy clustering methods in estimating the amount of tissue mixing in each voxel was evaluated by Brandt and Kharas [4] using dual-echo scan sequences. All of these approaches require vector valued images and tend to be computationally intensive.

Santago and Gage [5] have incorporated a model of PV effects to the statistical analysis of single-echo or scalar valued images, but they did not consider spatial information. More recently, Röll et al [1] presented a correction method for PV effects in scalar valued images based on the geometry and grey level intensity of thresholded objects. This method has no adjustable parameters and its accuracy has already been evaluated [6], but so far its application has been limited to objects extracted using intensity-based thresholding. The current paper presents a generalisation of this method and assesses its performance when applied to synthetic objects extracted using different segmentation strategies.

\section{Generalised Partial Volume Correction}

\subsection{Basic Concepts}

In MRI, the boundary voxels of an object are partly occupied by the object and partly occupied by the background. Let $V_{\text {true }}$ represent the true object volume and $V_{i n t}$ the volume of its interior voxels. We define the Geometric Partial Volume $V_{g P V}$ as the volume occupied by the boundary voxels. $V_{g P V}$ is given by $V_{\text {true }}-V_{\text {int }}$ and is proportional to the number of boundary voxels or, similarly, to the surface area $s_{a r e a}$. Such a dependence has been tested by considering synthetic objects of known volume created by a thresholded distribution of Coulomb charges and by placing them on a grid made up of cuboidal voxels [1]. The results obtained have shown $V_{g P V}$ to increase linearly with respect to $s_{\text {area }}$ and to be essentially independent of shape for mostly convex objects. Therefore, it is possible to estimate $V_{g P V}$ for a given object by considering a sphere of the same surface area sampled at a similar voxel size. However, $V_{g P V}$ is also highly dependent on the location of the object boundary on the sampling grid and on 
the geometry and resolution of the sampling grid. These two factors effectively determine the degree of partial object 'occupancy' or 'filling' of the boundary voxels and can be used to estimate a probability distribution of degrees of partial filling of boundary voxels $\left(p_{f i l l}\right)$ by randomly placing a sphere of equivalent $s_{\text {area }}$ in a cuboidal grid and counting the degrees of filling of its boundary voxels. $V_{g P V}$ is then assumed to add up according to $p_{f i l l}(x)$, where the contribution of each degree of filling $x$ is given by $f(x)=c_{1} x p_{f i l l}(x)$, with $c_{1}$ chosen so that $\int_{0+}^{1-} f(x) d x=V_{g P V}$. In order to obtain the partial volume overestimated by counting almost filled voxels as fully occupied, the complementary function $\tilde{f}(x)=c_{2}(1-x) p_{f i l l}(x)$ is also required. $\tilde{f}(x)$ expresses how each degree of filling $x$ contributes to a $V_{g P V}$ made up by the not filled boundary voxels and $c_{2}$ is such that $\int_{0+}^{1-} \tilde{f}(x) d x=V_{g P V}$.

Based on the above definitions, the following equation summarises the partial volume correction for an object that has been extracted using the minimum intensity threshold $I_{m i n}$ :

$$
V_{\text {corr }}=\sum_{I_{L}=I_{\text {min }}}^{I_{L}} p_{I}\left(I_{L}\right) \cdot\left(c_{1} \sum_{i=0}^{t_{\text {bin }}} x(i) p_{f i l l}(i)-c_{2} \sum_{i=t_{\text {bin }}}^{99}(1-x(i)) p_{f i l l}(i)\right)
$$

$p_{I}\left(I_{L}\right)$ is the probability distribution of intensities for the interior voxels. $x(i)$ denotes the degree of partial filling $i$ and $t_{b i n}$ indicates which of the bins (0-99) in the interval corresponds to the cut-off point of degree of filling $t$ determining the minimum degree of filling required to consider a boundary voxel as part of the object. For objects extracted using intensity thresholding $t$ is given by:

$$
t=\frac{\left(I_{\min }-\overline{I_{b g}}\right)}{\left(\overline{I_{L}}-\overline{I_{b g}}\right)}
$$

$\overline{I_{L}}$ is the mean grey level intensity of the lesion interior voxels and $\overline{I_{b g}}$ corresponds to the mean background grey level intensity.

$V_{\text {corr }}$ in Eq. 1 will be zero when a cut-off $t$ is selected for which the cumulative distribution of $p_{f i l l}$ below and above it are equal. Such a cut-off is regarded as 'optimal' $\left(t_{\text {opt }}\right)$ because the volume obtained from the segmentation $\left(V_{\text {seg }}\right)$ will be as close as possible to $V_{\text {true }}$. The value of $t_{\text {opt }}$ depends on the shape of $p_{f i l l}$ but, for a symmetric distribution it will always be 0.5 , i.e. halfway between object and background.

Of all the terms in Eq. 1, only $t_{b i n}$ is defined based on the intensity threshold $I_{\min }$ used to extract the object (Eq. 2). However, $p_{f i l l}$ is related to $V_{g P V}$, which in turn is obtained using the estimated $s_{a r e a}$. If the object has been extracted using intensity thresholding, $s_{\text {area }}$ is also related to the selected intensity thresholds as it is calculated from the resulting segmented object. Therefore, in order to use Eq. 1 with other segmentation methods, it is necessary to modify the determination of the cut-off point of degree of filling $t$ and the surface area $s_{\text {area }}$. 


\subsection{Cut-off Point of Degree of Filling}

Based on the definition of PV effect as the averaging of grey level intensities between the lesion interior and the background, $t$ essentially depends on the estimation of $I_{L}$ and $I_{b g}$. As $s_{a r e a}$ and $V_{g P V}$ have been estimated for the complete object, $t$ must also be globally computed. Thus, the mean of both interior and background grey level intensities can still be used, but the minimum threshold $I_{\min }$ will be replaced by the minimum grey level intensity of the lesion interior voxels $I_{L \min }$. This results in the following new cut-off point of degree of filling $t_{n}$ :

$$
t_{n}=\frac{\left(I_{L \min }-\overline{I_{b g}}\right)}{\left(\overline{I_{L}}-\overline{I_{b g}}\right)}
$$

This substitution makes the new cut-off point $t_{n}$ independent of the segmentation method. However, the implications of such substitution must be analysed. If the object is extracted using an intensity threshold method, then $I_{L m i n}=$ $I_{\text {min }}$. However, if the method employed was other than intensity thresholding, this equality is not necessarily true. Typically, not all of the lesion voxels obtained by the threshold method will be included (Fig. 1). This means that voxels with a grey level intensity in the range of those of the lesion interior could be present in the background. Due to the connectivity requirement for the objects of interest, these voxels would have been considered as boundary voxels of the thresholded object and not as part of the background. Hence, they should not be taken into account by the background estimation procedure as they actually comply with the required percentage of partial filling, but may have been excluded due to the segmentation method employed, or for perceptual or anatomical reasons.

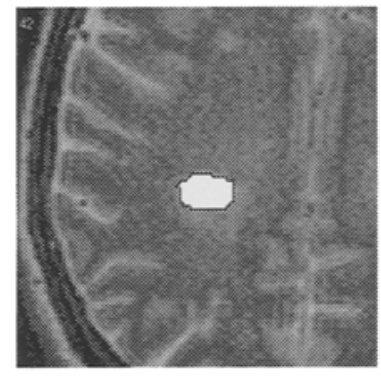

(a) Manual-MO.

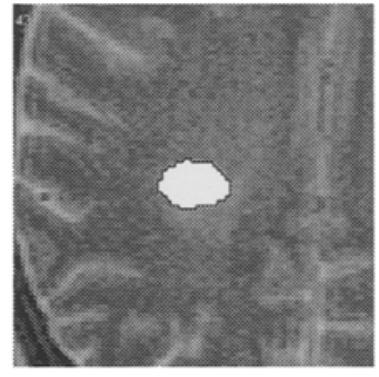

(b) Threshold-TRG.

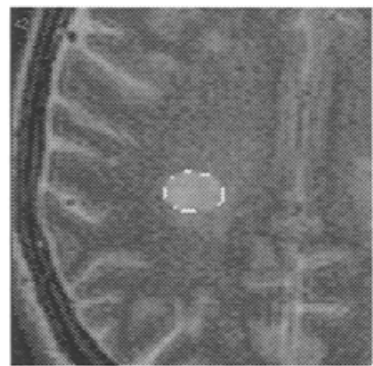

(c) TRG minus MO.

Fig. 1. Objects obtained by manual and threshold segmentation $\left(I_{\min }=I_{L \min }\right)$.

\subsection{Background Estimation}

In order to estimate the mean background intensity $\overline{I_{b g}}$ more accurately, the 3-D object morphology is considered by employing morphological operations to obtain a layer of background voxels $\mathbf{B}$ that fully surrounds it. The boundary voxels and those voxels corresponding to the background on both adjacent slices in the scanning plane are excluded (Fig. 2). Because it is common to find MS 
lesions close to structures with similar grey level intensities (e.g. ventricles), a thickness of only 2 voxels was used for $\mathbf{B}$.

According to Eq. 3 and based on the layer of voxels $\mathbf{B}$, the average background grey level intensity $\overline{I_{b g}}$ used to estimate $t_{n}$ is defined as:

$$
\overline{I_{b g}}=\frac{1}{m} \sum_{\forall I_{\mathbf{B}}} T\left(I_{\mathbf{B}}\right)
$$

$I_{\mathrm{B}}$ represents a grey level intensity in $\mathrm{B} . T\left(I_{\mathrm{B}}\right)=I_{\mathrm{B}}$ for $I_{\mathrm{B}}<I_{L m i n}$ and $T\left(I_{\mathbf{B}}\right)=0$ for $I_{\mathbf{B}} \geq I_{L m i n}$, and $m$ denotes the cardinality of the set $\left\{T\left(I_{\mathbf{B}}\right) \mid\right.$ $\left.I_{\mathrm{B}}<I_{L m i n}\right\}$. Fig. 3 illustrates the voxels considered for the estimation of $\overline{I_{b g}}$ using the background layer of voxels presented in Fig. 2 .

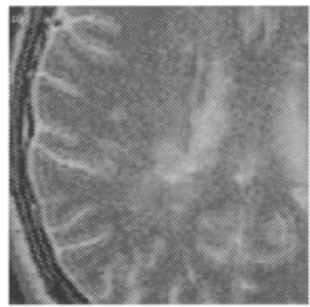

(a) Adjacent.

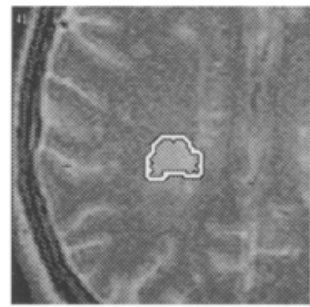

(b) First Slice.

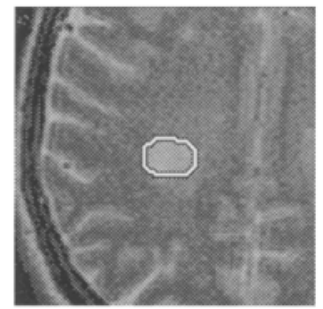

(c) Second Slice.

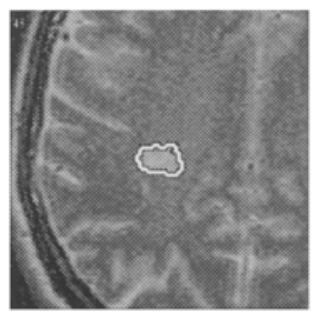

(d) Third Slice.

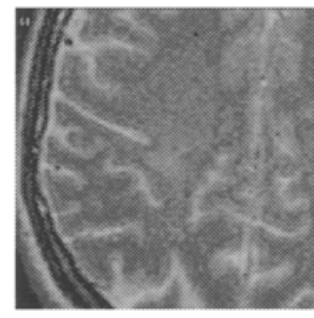

(e) Adjacent.

Fig. 2. Layer of background voxels $B$ for the object in Fig. 1.a.

\subsection{Geometric Partial Volume Interpolation}

When the PV correction is applied, the surface area and geometric partial volume at the desired $t_{n}$ are not known. Therefore, it is necessary to use the $s_{\text {area }}$ and $V_{g P V}$ of the segmented object. To minimise the effects of this circularity we obtained a new estimate of the surface area $\tilde{s}_{\text {area }}$ based on the calculated $t_{n}$ by considering an inner geometric partial volume $V_{I g P V}$ and an outer geometric partial volume $V_{O g P V}$. As before, $V_{I g P V}$ and $V_{O g P V}$ have been estimated by placing synthetic objects on a grid made up of cuboidal voxels, but in this case a cut-off point of degree of filling of 1.0 and 0.00001 were respectively used. $V_{I g P V}$ indicates the maximum possible underestimation as only completely filled boundary voxels are considered, whereas the maximum possible overestimation is given by $V_{O g P V}$. These two bounds are then used to estimate an inner surface 
area $s_{\text {Iarea }}$ and an outer surface area $s_{\text {Oarea }}$. Their usage results in a new approximation to the surface area given by $\tilde{s}_{\text {area }}=s_{\text {Iarea }}+t_{n} \cdot\left(s_{\text {Oarea }}-s_{\text {Iarea }}\right)$. $V_{I g P V}$ and $V_{O g P V}$ are also employed to compute new normalisation constants $c_{1 n}$ and $c_{2 n}$ such that $\int_{0+}^{1-} f(x) d x=V_{I g P V}$ and $\int_{0+}^{1-} \tilde{f}(x) d x=V_{O g P V}$.

$\tilde{s}_{\text {area }}$ is still dependent on the surface area of the segmented object as $s_{\text {area }}$ is initially used to estimate $V_{I g P V}$ and $V_{O g P V}$, but in our tests it has proven to be a much better estimate of the real surface area of the object.

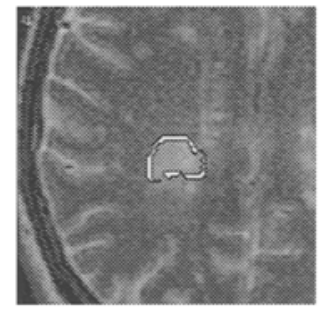

(a) First Slice.

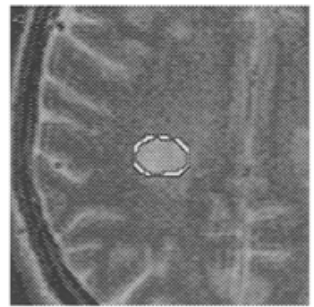

(b) Second Slice.

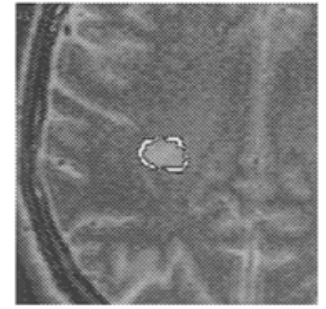

(c) Third Slice

Fig. 3. Voxels of $\mathbf{B}$ from Fig. 2 used to estimate $\overline{I_{b g}}$.

\section{Volume Estimation of Synthetic MS Lesions}

The synthetic lesions used to evaluate the proposed generalised PV correction method where generated by taking intensity distributions from a set of 'training' lesions of MS patient data and fusing them into a geometrically tractable model of lesion shape. This model was then implanted into the image of a healthy volunteer acquired with the same pulse sequence. We used radially parametrised intensity distributions as proposed in [6] to capture the intensity distribution within the lesion. The shape model was an ellipsoid with well-defined boundaries, thus providing a known true lesion volume $V_{t r u e}$. However, the boundaries of the implanted lesions are not sharply defined due to the radial intensity distribution approaching the background intensity $\overline{I_{b g}}$ towards the lesion borders and to the PV averaging of the lesion boundary voxels (Fig. 4).

\subsection{Segmentation Methods}

The segmentation methods employed to extract the synthetic lesions were: intensity thresholding and region growing (TRG), manual outlining (MO) and hierarchical segmentation (HS). The first method requires the selection of a minimum and maximum threshold, followed by the placement of a seed which is then used to obtain all connected voxels lying in the specified range of intensities. In manual outlining the operator uses free-hand drawing facilities to trace the boundary of the object. Lastly, the hierarchical segmentation is an agglomerative clustering algorithm that generates a hierarchy of sub-regions based on edge strength and edge stability [7]. The desired object is obtained by searching the hierarchy for a region complying with the constraints given by the user. 


\subsection{Volume Estimation and Correction}

The volume of 10 synthetic lesions implanted on high resolution $(0.9 \times 0.9 \times 2.0$ $\mathrm{mm}) T_{2}$ and FLAIR ( 5 each) MR scans of a healthy volunteer were estimated from the lesions extracted with the above segmentation methods. For manual outlining and hierarchical segmentation, the lesion extraction was performed twice by the same operator in order to observe the effect of their intrinsic variability on the volume correction. Table 1 presents the average of the absolute values of the relative errors of volume measurements without PV correction (UN) $\epsilon_{u n}=\left(V_{s e g}-V_{\text {true }}\right) / V_{\text {true }}$ and with PV correction (CO) $\epsilon_{c o}=$ $\left(V_{\text {seg }}+V_{\text {corr }}-V_{\text {true }}\right) / V_{\text {true }}$ as a percentage for each method and scan sequence.

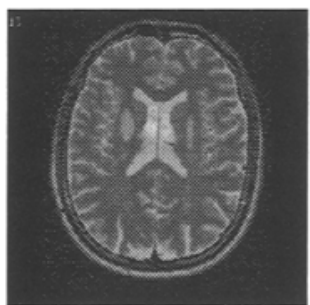

(a) $T_{2}$-weighted.

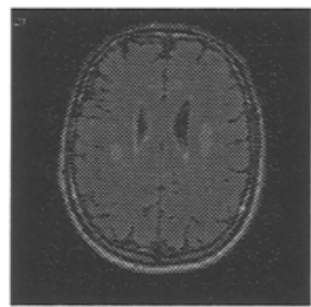

(b) FLAIR.

Fig. 4. Sample implanted lesions using the model proposed in [6].

\section{Discussion}

On the $T_{2}$ scans, only for one lesion the relative error of the corrected estimate was consistently larger. This was attributed to its small size and its closeness to a region with a very similar intensity that resulted in a poor estimation of $I_{L}$ and $I_{b g}$. On FLAIR, some of the implanted lesions also showed an increased relative error for the corrected volume estimates, particularly for the hierarchical segmentation. In general, the reduced contrast between lesion and background on FLAIR resulted in a less accurate segmentation and estimation of $I_{L}$ and $I_{b g}$. As the proposed PV correction method does not modify the extracted boundaries, but rather it estimates a volume correction assuming that they are as close as possible to the originals, a less accurate segmentation is likely to affect its performance.

Table 1 shows that, on average, the corrected volume estimate results in a smaller relative error than the uncorrected measurement. Manual outlining has the average largest uncorrected relative error while the other two methods present a very similar uncorrected relative error. This might be due to a greater operator dependence and subsequent variability. Overall, however, the PV correction for the manual outlining and hierarchical segmentation result in a larger reduction of the relative error. 


\begin{tabular}{|c||l|l|l|l|l|l|}
\hline Scan & $\begin{array}{l}\text { TRG } \\
\text { (UN) }\end{array}$ & $\begin{array}{l}\text { TRG } \\
\text { (CO) }\end{array}$ & $\begin{array}{l}\text { MO } \\
\text { (UN) }\end{array}$ & $\begin{array}{l}\text { MO } \\
\text { (CO) })\end{array}$ & $\begin{array}{l}\text { HS } \\
\text { (UN) }\end{array}$ & $\begin{array}{l}\text { (CO) } \\
\text { (CO }\end{array}$ \\
\hline \hline$T_{2}$ & 13.8 & 12.3 & 16.4 & 9.4 & 13.3 & 9.6 \\
\hline$\overline{F L}$ & 13.8 & 13.2 & 18.7 & 11.7 & 13.4 & 12.4 \\
\hline
\end{tabular}

Table 1. Average of the absolute values of the relative error for each scan sequence and segmentation method presented as percentage.

\section{Conclusions and Future Work}

In this paper we have presented a generalisation of the geometry and intensity based partial volume correction originally proposed in [1]. This generalisation was evaluated by using synthetic lesion implants on high resolution $T_{2}$ and FLAIR MR scans which were subsequently extracted by three different segmentation methods. On average, the relative error of the corrected volume estimates showed a decrease when compared to that of the uncorrected estimates. Further evaluation of the method is now required to more thoroughly study its combination with the different segmentation methods, as well as to fully validate the proposed generalisation.

Among the future work being contemplated is a background estimation procedure based on the discrimination of the surrounding tissue classes in order to deal with the problem of lesions close to the ventricles or other areas with a similar grey level intensity. The determination of a local degree of filling is also being considered, but its usage would imply local estimation of a valid $V_{g P V}$ and $p_{f i l l}$, which is by no means a trivial task.

It must be said that some of the considerations presented in this paper are only valid for mostly convex objects with no inside holes, which is the case of most MS lesions. For more complicated objects these considerations will have to be re-evaluated.

\section{References}

1. S.A. Röll, A.C.F. Colchester, P.E. Summers and L.D. Griffin. Intensity-Based Object Extraction from $3 D$ Medical Images Including a Correction for Partial Volume Errors. BMVA Press, 1994. In: Proc. BMVC 1994, pp 205-214.

2. H. Soltanian-Zadeh, J.P. Windham and A.E. Yagle. Optimal Transformation for Correcting Partial Volume Averaging Effects in Magnetic Resonance Imaging. IEEE TNS vol. 40 no. 4, pp 1204-1212, 1993.

3. H.S. Choi, D.R. Haynor and Y. Kim. Partial Volume Tissue Classification of Multichannel Magnetic Resonance Images-A Mixel Model. IEEE TMI vol. 10 no. 3, pp 395-407, 1991.

4. M.E. Brandt and Y.F. Kharas. Simulation Studies of Fuzzy Clustering in the Context of Brain Magnetic Resonance Imaging. IEEE, 1993. In: Proc. 3rd Intl. Conf. on Industrial Fuzzy Control and Intelligent Systems IFIS'93, pp 197-203.

5. P. Santago and H.D. Gage. Quantification of MR Brain Images by Mixture Density and Partial Volume Modeling. IEEE TMI vol. 12 no. 3 , pp 566-574, 1993.

6. S.A. Röll, A.C.F. Colchester, L.D. Griffin, P.E. Summers, F. Bello, B. Sharrack and D. Leibfritz Volume Estimation of Synthetic Multiple Sclerosis Lesions: An Evaluation of Methods. SMR, 1995. In: Proc. 2nd Meeting SMR1995, pp 809.

7. L.D. Griffin, A.C.F. Colchester, S.A. Röll, C.S. Studholme Hierarchical Segmentation Satisfying Constraints. BMVA Press, 1994. In: Proc. BMVC 1994, pp 135-144. 